\title{
Charge reversal in cadmium sulfide in diffusion controlled pattern formation process in agar gel
}

\author{
N PALANIANDAVAR ${ }^{\dagger}, \mathrm{N}$ KANNIAH, F D GNANAM and \\ P RAMASWAMY* \\ Crystal Growth Centre, Anna University, Madras 600025 , India \\ ${ }^{\dagger}$ Government Arts College, Madras 600035 , India
}

MS received 26 September 1984; revised 9 Feburary 1985

\begin{abstract}
Investigations were carried out to obtain the experimental conditions for the formation of a diffusion controlled revert pattern followed by a direct spatial paltern of bands in agar gel. The formation of revert to direct periodic pattern in the same tube is explained on the basis of adsorption, electrical double layer and charge reversal. On the basis of DLvo and Shinohara's theory, surface potential is calculated.
\end{abstract}

Keywords. Pattern formation; cadmium sulfide; adsorption; flocculation; charge reversal; agar gel.

\section{Introduction}

The precipitation of weakly soluble salts in aqueous solution is produced by diffusion of one of the constituent ions into an initially homogeneous solution containing another ion necessary for precipitation. Depending on the parameters, well defined visible bands of precipitate divided by apparently clear zones are formed. The pattern formation was first observed by Liesegang (1896) in a silver chromate system in gelatin. The interspacing between successive bands of precipitate increases as the order of the band from the interface increases. This type of pattern formation is popularly known as the direct type of Liesegang bands (Gnanam et al 1980; Muller et al 1982). A few substances like silver chromate, ferricyanides of ferrous, nickel, cobalt and copper (Mathur and Ghosh 1958) have rarely been precipitated in a different manner in which the intermittent space between successive bands decreases as the order of the band increases and this formation is known as revert bands. Hedges and Henley (1928), Mukherjee and Chatterji (1930), Packter (1955), von Hook (1937) and Kanniah et al (1981), have obtained such revert patterns of various substances in gel media.

Singji et al (1971) and Hatschek (1925) have observed direct spatial patterns of precipitation of cadmium sulfide in gelatin. Periodic precipitation of cadmium sulfide was obtained by Daus and Tower (1929) by mixing sodium sulfide of 0.4 to $1 \mathrm{~N}$ with the gel and having 0.8 to $2 \mathrm{~N}$ of cadmium chloride as supernatent electrolyte. In this article we describe a unique observation made by us in cadmium sulfide which exhibits a spatial periodic pattern of revert followed by the direct system in the same tube. Kanniah et al (1981) have reported similar observation for silver iodide sol. But none of them have

\footnotetext{
* To whom all correspondence should be addressed
} 
observed revert to direct in the same tube for a semiconducting substance like cadmium sulfide. The experimental conditions for conversion of patterns from revert to direct periodic bands have been investigated. An attempt has been made to explain this phenomenon on the basis of adsorption, charge reversal and flocculation.

\section{Experiment}

Agar gel of $0.6 \%$ by weight has been prepared as reported by Gnanam et al (1980). Varying amounts of cadmium chloride ranging from 0.004 to $0.01 \mathrm{M}$ were dissolved in agar and $\mathrm{pH}$ adjusted in the range 1.5-2.14 by hydrochloric acid (Palaniandavar et al 1984). The outer electrolyte, ammonium sulfide solution of concentration 0.734 to $2.935 \mathrm{M}$, was taken over the set gel and a revert pattern observed. When the $\mathrm{pH}$ of the gel impregnated with $0.005 \mathrm{M}$ of cadmium chloride was 1.38 and the concentration of the outer electrolyte ammonium sulfide $1.467 \mathrm{M}$, revert followed by direct spatial pattern was observed. The position of the bands from the gel boundary was measured by cathetometer.

\section{Results and discussion}

When the supernatant electrolyte ammonium sulfide, which is many times greater in concentration than the innner electrolyte cadmium chloride, diffuses into the gel impregnated with cadmium chloride, cadmium sulfide sol is formed as per the irreversible reaction

$$
\mathrm{CdCl}_{2}+\left(\mathrm{NH}_{4}\right)_{2} \mathrm{~S} \rightarrow \mathrm{CdS}+2 \mathrm{NH}_{4} \mathrm{Cl} \text {. }
$$

Cadmium sulfide sol is negatively charged owing to the preferential adsorption of sulfide ion (Weiser 1938; Sen 1925). In addition to cadmium sulfide sol, the strong electrolyte ammonium chloride is also formed as a parasitic electrolyte. As more and more outer electrolyte diffuses, the sol front advances, spreading the sol region, and thereby increases the ionic environment. Thus the electrical double layer formed around the colloid particle is crowded with sulfide, chloride and ammonium ions. As the ionic concentration reaches a characteristic value, the electrical double layer is compressed and $\zeta$ potential is reduced to a minimum and flocculation is triggered.

According to Shinohara's theory (1970) the characteristic concentration which is responsible for flocculation can be given as

$$
\Gamma=C_{10}\left\{1-\left[G\left(k_{1}\right) / G(k)\right]\right\},
$$

where $C_{10}$ is the concentration of ammonium sulfide,

$$
\begin{aligned}
G(\beta) & =\left(1 /(2 \pi)^{1 / 2}\right) \int_{0}^{\beta} \exp \left(-\frac{1}{2} \eta^{2}\right) \mathrm{d} \eta \\
\beta & =k \text { or } k_{1}, k=k / p \text { and } p=\left(x_{n+1} / x_{n}\right),
\end{aligned}
$$

where $x_{n}$ is the position of the $n$th band from the gel boundary, $p$ is known as spacing coefficient and $k$ is called the front constant which can be estimated using Adair's equation (1970). 
Due to the imposed concentration gradient of the outer electrolyte, the surface potential $\left(\Psi_{0}\right)$ progressively decreases from the gel boundary and hence the zeta potenial is lowered below a critical value which allows the agglomeration of particles into aggregates that precipitate out. The lowering of zeta potential is effected by contraction of the double layer by specific adsorption of hydrolysed ammonium ion (Yoder et al 1980; Napper and Hunter 1975). Hence, when the concentration of ammonium ions attains a critical value, the negatively charged cadmium sulfide sol is flocculated and the first band is formed.

As diffusion proceeds further downwards, the surface potential and hence zeta potential progressively decreases leading to a decrease in the flocculation value (Voyutsky 1978). Thus bands are successively formed and the interspacing decreases resulting in the formation of revert periodic structure.

The relation connecting the surface potential $\Psi_{0}$ and the characteristic concentration $\Gamma$ at which flocculation is triggered may be derived on the basis of the DerjaguinLandau-Verwey-Overbeek theory of the stability of colloids (Vervey and Overbeek 1948). The sol particles are assumed to be comparatively large and flat faced so that they may be approximated as flat plates. The total potential energy of interaction between the sol particles is

$$
V=(6 \pi c k T / \alpha) \gamma^{2} e^{2 k \delta}-\left(A / 48 \pi \delta^{2}\right)
$$

where $c$ is the concentration of the electrolyte per cubic centimeter,

$$
\begin{aligned}
& \alpha=\left[8 \pi c z^{2} e^{2} / \xi k T\right]^{1 / 2}, \\
& \gamma=\left[\exp \left(z e \Psi_{0} / 2 k T\right)-1\right] /\left[\exp \left(z e \Psi_{0} / 2 k T\right)+1\right],
\end{aligned}
$$

$A$-Hamker's constant, $\delta$-half the distance between the particles, $\xi$-dielectric constant, $k$-Boltzmann constant, $z$-valency.

$$
\text { For flocculation } \frac{\mathrm{d} V}{\mathrm{~d} \delta}=0
$$

Differentiating (4) and applying the condition for flocculation, we can obtain characteristic concentration for flocculation as

$$
\Gamma=\left(8 \times 10^{-33} \Psi_{0}^{4}\right) /\left(A^{2} z^{2}\right) \mathrm{m} \mathrm{mol} \mathrm{dm}^{-3},
$$

where $\Psi_{0}$ is expressed in millivolts.

From the elementary double layer theory, the surface charge $\sigma_{0}$ can be calculated using the equation

$$
\Psi_{0}=0.05 \times 10^{3} \sinh ^{-1}\left[\sigma_{0} / 11 \cdot 74(\Gamma)^{1 / 2}\right]
$$

Due to continuous downward diffusion of sulfide ion from the interface, the concentration decreases gradually and the rate of decrease of potential with distance increases more rapidly (Krylov and Levich 1963); a stage is reached at which the charge of the sol is neutral. This represents the isoelectric region which indicates a transition from the revert to the direct system.

Below the transition zone the concentration of sulfide ion is very low and the sulfide ion undergoes hydrolysis $\mathrm{S}^{2-}+\mathrm{H}_{2} \mathrm{O} \rightarrow \mathrm{HS}^{-}+\mathrm{OH}^{-}$(Moeller and Connor 1972). Hence the effective charge on the surface is reversed to positive (Anderson and Rubin 1981). The concentration of cadmium chloride is uniform throughout the gel and is also complexed as $\mathrm{CdCl}^{+}, \mathrm{CdCl}_{2}$ and $\mathrm{Cd}^{2+}$ (Mills and Hertz 1982; Hamer 1959). In 
addition to this, ammonium ion causes a significant enhancement in their adsorptivity and consequently influences the reversal of negatively charged sol to positively charged sol (Shaw 1970). On account of the positive charge of the sol, the potential of the particle is now positive and the sulfide ion acts on the diffused portion of the double layer. More and more sulfide ion is required to compress the double layer to produce flocculation. Hence the first band is formed at a point at which the characteristic concentration is reached after transition.

After the formation of the first band, the positively charged cadmium sulfide sol is formed as before. More and more sulfide ion has to diffuse for the onset of flocculation. Therefore, the next band is formed at a greater distance with successively increasing interspace leading to the formation of direct periodic bands. The colour of the band is light white-yellow which can be attributed to the inclusion of chloride ions.

The characteristic concentration $\Gamma$ of the outer electrolyte to initiate flocculation has been computed from Shinohara's theory. The extrapolation (figure 1) between $\Gamma$ and the position of the band shows the trend of decrease of $\Gamma$ in the negatively charged sol region and then the increase in the positively charged direct pattern region. Figure 2 gives the relationship between the surface potential and the distance of the bands from the gel boundary. The portion $\mathrm{AB}$ in figure 2 represents the revert spatial structure and $\mathrm{CD}$ the direct structure. Whenever the surface potential is low, the sol is least stable (Lyklema 1977). The flocculation value is much less. The portion BC denotes this transition region where the surface potential decreases, attains zero at the point $M$ and then increases in the positive region. The point $M$ represents zero point charge which is the transition point for cadmium sulfide.

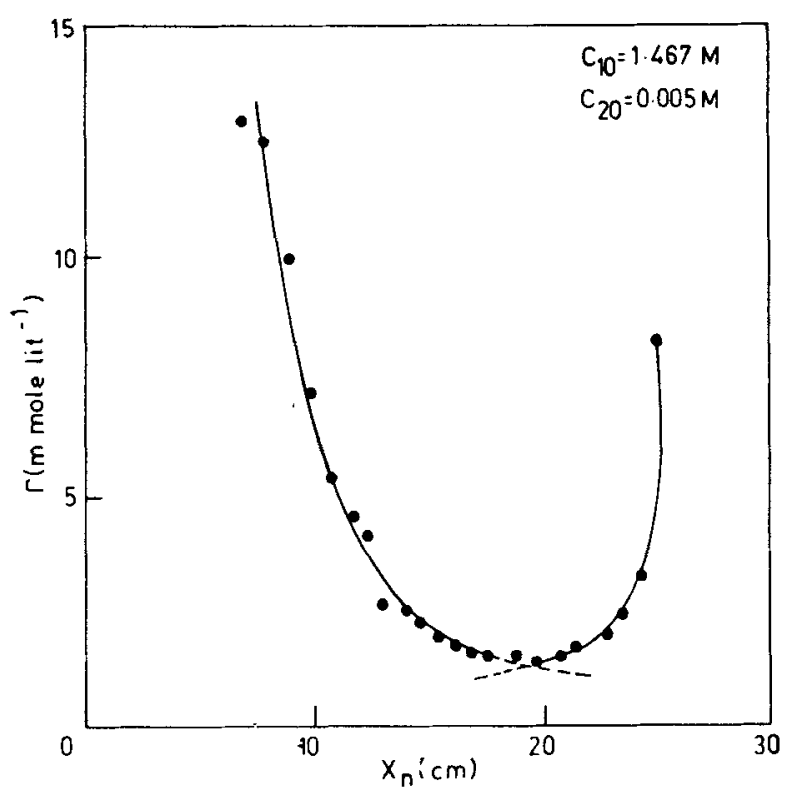

Figure 1. The flocculation value (calculated) os the position of the band. 


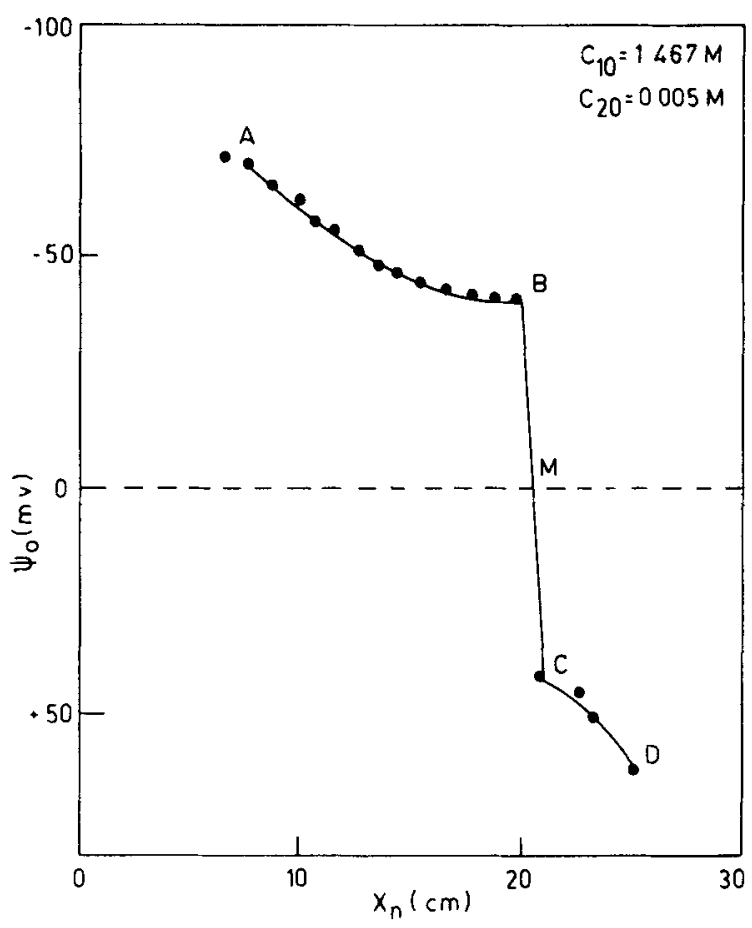

Figure 2. Dependence of surface potential (calculated) on the position of the band from the gel boundary.

\section{Conclusion}

The reversal of charge of cadmium sulfide is explained on the basis of adsorption and hydrolysis. The formation of revert to direct is due to the reversal of charge from negative to positive.

\section{References}

Anderson M A and Rubin A J 1981 Adsorption of inorganics at solid liquid interfaces (Ann Arbor Collingwood: The Butterworth Group Science Publishers) pp. 34, 43, 106

Daus V and Tower O F 1929 J. Phys. Chem. 33605

Gnanam F D, Krishnan S, Ramasamy P and Laddha G S 1980 J. Colloid Interface Sci. 73193

Hamer W J 1959 The structure of electrolytic solutions (New York: John Wiley) p. 360

Hatschek E 1925 Kolloid-Z. 37297

Hedges E S and Henley R V 1928 J. Chem. Soc. 2714

Kanniah N, Gnanam F D, Ramasamy P and Laddha G S $1981 \mathrm{~J}$. Colloid Interface Sci. 80369

Krylov V S and Levich V C 1963 Russ. J. Phys. Chem. 371224

Liesegang R E.1896 Naturwiss. Wochenschr. 11353

Lyklema 1977 Trends in electrochemistry (New York: Plenum)

Mathur P B and Ghosh S 1958 Kolloidn. Zh. 159143

Mills R and Hertz H G 1982 J. Chem. Soc. Faraday Trans. I 783287

Moeller T and Connor R O 1972 Ions in aqueous systems (New York: McGraw Hill) p. 109

Mukherjee L N and Chatterji A C 1930 Kolloidn. Zh. $\mathbf{S 0} 147$ 
Muller S C, Kai S and Ross J 1982 J. Chem. Phys. 864078

Napper D H and Hunter J 1975 Hydrosols, surface chemistry of Colloids; Physical Chemistry Series I (London: Butterworths) (ed) P Kerker Vol. 7 pp. 293-295

Packter A 1955 Kolloidn. Zh. 142109

Palaniandavar N, Gnanam F D and Ramasamy P 1984 J. Chem. Phys. 803445

Sen K C 1925 J. Phys. Chem. 291540

Shaw D J 1970 Introduction to colloid surface chemistry (London: Butterworths) p. 134

Shinohara S 1970 J. Phys. Soc. Jpn. 291073

Singji H, Kumar R, Kant B M and Kant K 1971 Proc. Natl. Acad. Sci. India A41

Verwey J N and Overbeek J Th G 1948 Theory of stability of lyophobic colloids (New York: Elsevier) pp. 7, 118 von Hook 1937 J. Phys. Chem. 41593

Voyutsky S 1978 Colloid chemistry (Moscow: Mir Publishers)

Weiser H B 1938 Inorganic colloid chemistry (New York: John Wiley), Vol. 3, p. 260

Yoder C H, Snydam H and Snavely A 1980 Chemistry (New York: Harcourt Brace Jovanovich) p. 566 